\title{
Bilan d'un essai de contrôle de Biomphalaria glabrata en Guadeloupe à l'aide d'un Trématode stérilisant
}

\author{
par H. NASSI *, J.-P. POINTIER ** et Y.-J. GOLVAN *** \\ * Département de Biologie animale (Dir.: Pr C. Combes), \\ Université, F 66025 Perpignan Cedex. \\ * Laboratoire de Biologie marine et de Malacologie (Dir.: Pr B. Salvat), \\ Ecole Pratique des Hautes Etudes, 55, rue de Buffon, F 75005 Paris. \\ *wak Laboratoire de Parasitologie, Faculté de Médecine Saint-Antoine, \\ 27, rue de Chaligny, F 75571 Paris Cedex 12.
}

\section{Résumé.}

Un essai de contrôle biologique de Biomphalaria glabrata a été réalisé en Guadeloupe à l'aide de Ribeiroia marini guadeloupensis Nassi, 1978, Trématode autochtone qui stérilise le Mollusque vecteur de Schistosoma mansoni. L'essai s'est déroulé dans une mare dont la population de $B$. glabrata présente un cycle démographique annuel lié à l'alternance saison sèche-saison humide. Environ 8 millions d'œufs de $R$. marini ont été introduits en 15 mois dans la mare. Malgré le faible rendement de ces introductions, dû notamment à la forte turbidité de l'eau, cet essai a été positif puisqu'il s'est soldé par la disparition de la quasi-totalité des Mollusques. L'analyse des résultats concernant l'évolution des effectifs de B. glabrata et l'évolution de la prévalence montre qu'une efficacité comparable pourrait être obtenue par des déversements d'œufs limités dans le temps mais massifs.

\section{Summary.}

Evaluation of a trial to control Biomphalaria glabrata in Guadeloupe by using a sterilizing Trematode.

A trial of biological control of Biomphalaria glabrata was carried out in Guadeloupe by using Ribeiroia marini guadeloupensis Nassi, 1978, an autochtonal Trematode which sterilizes the snail vector of Schistosoma mansoni. The trial took place in a pond in which the population of $B$. glabrata presents an annual demographic cycle related to the alternation

Accepté le 18 décembre 1978. 
of dry and rainy seasons. About 8 millions eggs of $R$. marini were introduced in 15 months into the biotope. Despite the low yield of these introductions, due particularly to the high turbidity of the water, this trial gave positive results in the disappearance of almost all the snails. The analysis of the results concerning the evolution of the levels of the snails and of the prevalence shows that a comparable efficiency could be obtained by massive introductions of eggs during short periods.

\section{Introduction}

Des déversements massifs d'œufs de Trématodes dans des mares expérimentales ou naturelles ont déjà été réalisés ; ces déversements ont eu pour but de déterminer si l'antagonisme entre deux espèces de Trématodes à l'intérieur d'un Mollusque hôte pouvait constituer un moyen de réduire la prévalence de l'une de ces deux espèces, dite espèce cible, dans une population de ce Mollusque (Heyneman et Umathevy, 1967 ; Lie et coll., 1970, 1971 ; Lie et Owyang, 1973; Lie et coll., $1974 a, 1974 b$ ). L'essai réalisé en Guadeloupe et dont nous rapportons les résultats a été conçu dans une optique différente; lors de cet essai nous avons essayé d'évaluer l'impact d'une introduction massive d'œufs d'un Trématode stérilisant sur l'évolution démographique d'une population naturelle du vecteur de Schistosoma mansoni, Biomphalaria glabrata. Parmi les Trématodes qui parasitent et stérilisent B. glabrata en Guadeloupe (Golvan et al., 1975) notre choix s'est porté pour cet essai sur Ribeiroia marini guadeloupensis, pour les raisons déjà exprimées par l'un d'entre nous (Nassi, 1978).

\section{Matériel et méthodes}

Le site d'intervention est une mare de Grande-Terre (partie est de la Guadeloupe) ayant fait l'objet d'études quantitatives préliminaires au cours des années 1974-1975 (Pointier et al., 1977). Les données ainsi recueillies nous ont servi de références pour l'interprétation des résultats de notre essai. Cette mare présente un cycle hydrique annuel lié à l'alternance saison sèche-saison humide. Elle s'assèche presque totalement en été (juillet-août) ; sa remise en eau s'amorce fin août, début septembre ; sa surface est maximale en novembre (environ $380 \mathrm{~m}^{2}$ ), la profondeur maximale mesurée au centre étant de $1,20 \mathrm{~m}$. L'évolution de la population de $B$. glabrata au cours d'un cycle annuel est marquée par une explosion démographique intervenant lors de la remise en eau et qui amène la population à son maximum en octobre-novembre. Puis la population décline pendant toute la saison sèche, les survivants assurant le repeuplement de la mare à la saison des pluies suivante.

L'essai s'est déroulé de septembre 1976 à décembre 1977. L'évolution de la popu. lation de Mollusques a été suivie par des échantillonnages effectués tous les 15 jours. Jusqu'en juin 1977, la population totale a été évaluée à partir de prélèvements de $1 / 10 \mathrm{~m}^{2}$ effectués à l'aide d'un cylindre en tôle à raison de 10 prélèvements dans chacune des 3 principales strates végétales. Cette méthode qui a l'avantage de limiter les 
perturbations du milieu, a par contre tendance à sous-estimer les effectifs (1). A partir d'août 1977, étant donné la faible densité de Mollusques, nous avons été amenés à modifier notre méthode d'échantillonnage. Nous avons tenté d'estimer l'effectif de Mollusques en prélevant pendant 1 heure et 3 jours de suite le maximum d'individus possible. Il est certain que ces interventions répétées auxquelles nous avons été contraints ont perturbé le milieu (2). Quelle que soit la méthode employée, l'échantillonnage ne permet pas de récolter les nouveau-nés et sous-estime fortement les juvéniles. Les graphiques présentés ne prennent donc en compte que les individus d'un diamètre égal ou supérieur à $4 \mathrm{~mm}$.

Trois lots de 10 rats infestés par R. marini guadeloupensis (cf. Nassi, 1978) ont été utilisés successivement: le premier de septembre 1976 à février 1977, le second de février à juin 1977, le troisième d'août à décembre 1977. Tous les œufs produits ont été déversés dans la mare (nombre total estimé entre 7 et 9 millions). Jusqu'en juin 1977, la prévalence a été évaluée tous les 15 jours sur des échantillons d'importance variable correspondant à 1 heure de ramassage. Après août 1977, la prévalence a été déterminée à partir de tous les Mollusques récoltés. Les Mollusques sont conservés 24 heures au laboratoire. Les infestations patentes qui seules peuvent être ainsi décelées donnent le taux d'émission de la population («shedding rate » des auteurs anglo-saxons).

\section{Résultats}

\section{Evolution des effectifs de Mollusques (fig. 1 A).}

L'introduction du parasite commence le 4 septembre 1976. La remise en eau de la mare est déjà amorcée et la nouvelle génération apparaît dans les prélèvements environ un mois plus tard. L'explosion démographique se produit normalement et la population est à son maximum en octobre. Le début du mois de novembre est marqué par un effondrement démographique qui amène la population à un niveau inférieur à celui de l'année témoin. Le parasite apparaît donc capable de se comporter comme un agent pathogène efficace vis-à-vis d'une population jeune de Planorbes. Par la suite, le déclin se ralentit mais la mortalité va rester importante; en effet, les données quantitatives recueillies en 1974 et de nombreuses observations ponctuelles effectuées tant dans cette mare que dans d'autres montrent que l'assèchement s'accompagne d'une augmentation souvent considérable de la densité de Planorbes. En 1977, dans la mare étudiée, la mortalité est telle que c'est le phénomène inverse qui se produit (fig. 2). A la mi-juin, une récolte exhaustive ne nous livre que 30 individus et aucun fin juin.

(1) Cette sous-estimation a pu être mise en évidence dans une autre mare où cette méthode a été comparée à la méthode par capture-recapture dont la précision est beaucoup plus satisfaisante.

(2) La méthode de capture-recapture nous a montré qu'en procédant ainsi il n'était possible de récupérer qu'environ $1 / 4$ de la population. 


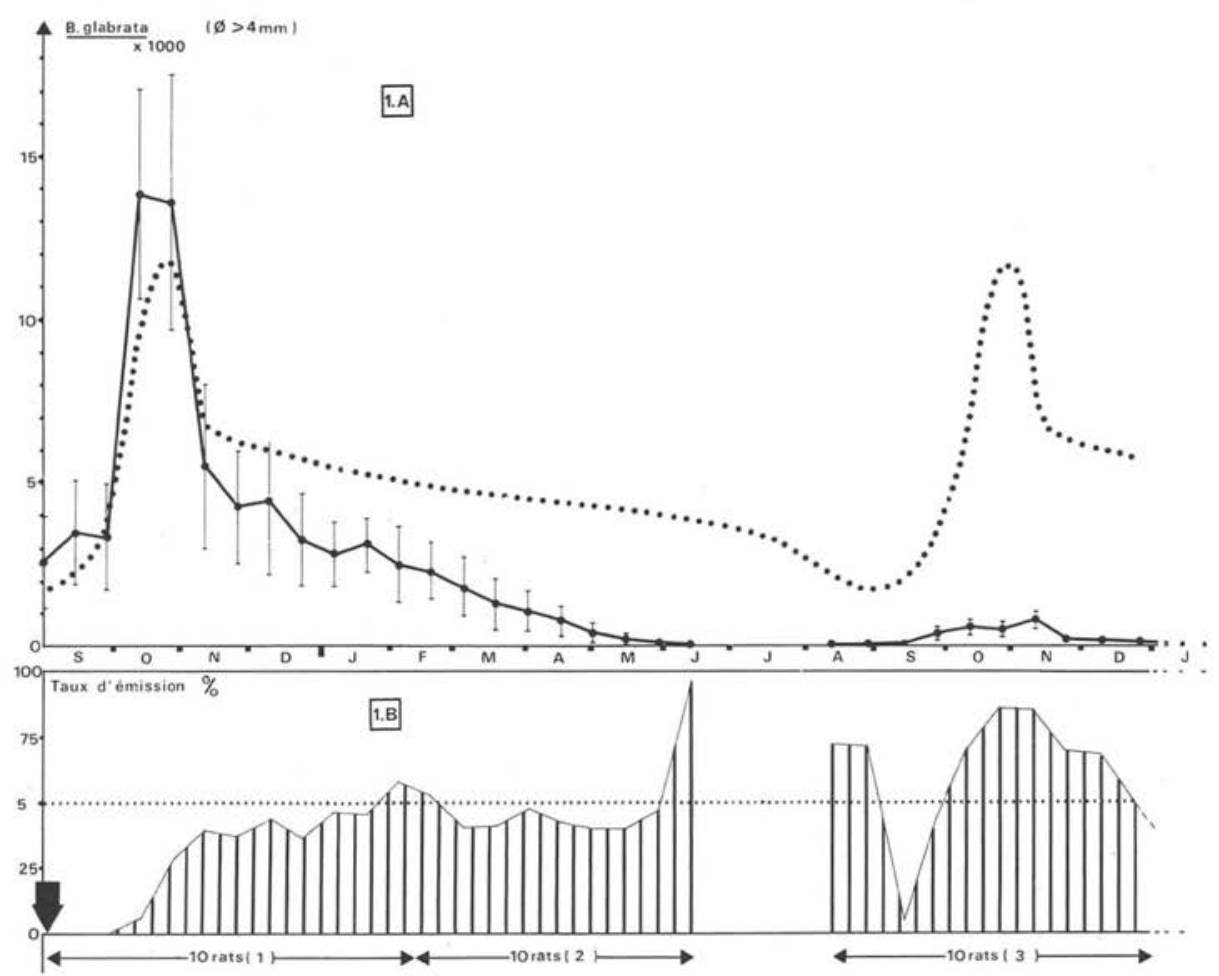

Fig. 1. A : évolution des effectifs de B. glabrata au cours de l'essai (trait plein) comparée à la courbe témoin établie en 1974-1975 (pointillés). B : évolution du taux d'émission pendant l'essai. La flèche indique le début des introductions d'œufs.

La remise en eau débute vers la mi-août. Les prélèvements révèlent la présence d'une faible population d'adultes (moins de 50 individus) dont les $2 / 3$ environ sont parasités. La nouvelle génération apparaît à la même époque que l'année précédente, mais son effectif maximum ne dépasse pas 800 individus. Cette nouvelle génération décline rapidement jusqu'en décembre 1977. Des échantillonnages ponctuels effectués durant le premier trimestre 1978 montrent que la population ne compte plus que quelques dizaines d'individus.

\section{Evolution de la prévalence (fig. $1 \mathrm{~B}$ ).}

Les premières émissions de cercaires sont observées le 11 octobre, soit environ 5 semaines après le premier déversement d'œufs dans la mare. Le taux d'émission est alors de $6,2 \%$. Au bout de 10 semaines, il atteint $40 \%$. La population vient de subir l'effondrement démographique signalé plus haut et la parasitose affecte toutes les classes de taille au-dessus de $4 \mathrm{~mm}$. Par la suite, le taux d'émission se stabilise à des valeurs le plus souvent proches de $40 \%$; il ne dépasse $50 \%$ qu'en février. Puis 
Fig. 2. Evolution de la densité de B. glabrata par $\mathrm{m}^{2}$ de février à juillet (saison sèche) pendant l'essai (trait plein) et pendant l'année témoin (pointillés).

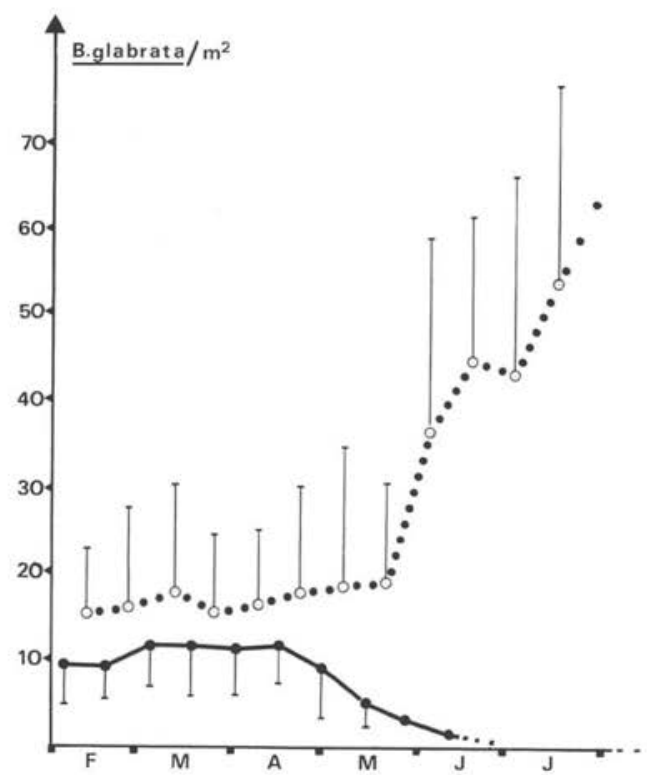

à la mi-juin, le dernier prélèvement révèle une forte augmentation de la prévalence. Sur les 30 Planorbes récoltés, 25 émettent dans les 24 heures qui suivent; sur les 5 individus négatifs, conservés à titre exceptionnel au laboratoire, 4 émettent au bout de 5 jours, révélant ainsi une infestation récente.

Le déversement des œufs est interrompu à ce moment-là. Il reprend avec l'élévation de niveau corrélative de la nouvelle saison des pluies alors que la population qui compte environ 50 individus est parasitée aux 2/3. Il n'y a donc dans la mare qu'une quinzaine de Mollusques capables de pondre. L'ancienne génération disparaît durant la première quinzaine de septembre; cette disparition se traduit par une chute brutale de la prévalence. L'impact sur la nouvelle génération est plus marqué qu'en 1976. La prévalence atteint $40 \%$ durant le mois de septembre, soit environ un mois et demi plus tôt qu'en 1976. De plus, il est visible que l'incidence reste forte pendant le mois d'octobre, puisque le taux d'émission dépasse $85 \%$ lors des échantillonnages de fin octobre et de mi-novembre. Puis le taux d'émission diminue avec le déclin de la population; il n'est plus que de $50 \%$ à la fin du mois de décembre. Le parasite est encore présent dans la mare en janvier 1978. En mars, aucun des rares Mollusques récoltés n'est infesté.

\section{Discussion}

L'introduction des œufs du Trématode stérilisant a été efficace puisqu'elle a amené la disparition quasi totale des Mollusques. Si l'on s'en tient à ce seul résultat, le bilan final de cet essai apparaît donc comme positif. 
En outre, bien que cet essai ait été réalisé dans un milieu de dimensions modestes et soumis à fluctuation saisonnière, il nous semble possible d'en déduire un certain nombre d'enseignements pour la poursuite de recherches en vue d'un contrôle biologique des vecteurs de Schistosomoses.

Une première déduction concerne l'efficacité des déversements d'œufs. Le fait que cette efficacité ait varié au cours de l'essai trouve en grande partie son explication dans les changements de structure de la population. C'est une population jeune qui subit en automne 1976 le premier impact du parasite; nous pensons que la rapidité avec laquelle le taux d'émission de $40 \%$ a été atteint est due pour l'essentiel à la réceptivité élevée des Mollusques jeunes. Par la suite, le vieillissement de la population avec comme conséquence une baisse de cette réceptivité a certainement contribué à stabiliser la prévalence de novembre 76 à mai 77 (3). D'autres facteurs ont sans doute concouru au maintien de cette stabilité. Il est probable par exemple que la mortalité plus importante des individus parasités a joué un rôle indirect en compensant l'apparition des cas nouveaux. On peut remarquer également qu'avec une prévalence voisine de $50 \%$, la probabilité de rencontre entre un miracidium et un Mollusque sain est diminuée de moitié. Cependant, avec l'assèchement de la mare et le fort déclin de la population, la densité de miracidiums et le nombre de miracidiums disponibles par Mollusque n'ont cessé d'augmenter. Ces deux paramètres ont atteint courant mai des valeurs suffisantes pour que les conditions d'infestation des individus âgés ayant jusque-là résisté soient réalisées. A l'automne 1977, avec le faible renouvellement démographique, le rapport nombre de miracidiums/nombre de Mollusques a été beaucoup plus favorable qu'en 1974. Ce fait explique à lui seul que la population jeune ait été parasitée à plus de $85 \%$.

La population s'est donc avérée plus vulnérable à deux moments de son cycle démographique: à l'automne lors de son renouvellement, et au printemps lorsque l'assèchement de la mare était déjà très marqué. Nous pensons que des interventions massives limitées à ces deux périodes auraient permis de parvenir à une réduction comparable de la population.

D'autres enseignements peuvent être tirés de cet essai en ce qui concerne le rendement de ces déversements d'œufs.

Lie et al. $(1974 b)$ ont mis l'accent sur un certain nombre de facteurs susceptibles de faire baisser le rendement de ce type d'essai : ensevelissement des œufs dans la vase, turbidité de l'eau qui réduit la capacité du miracidium à trouver son hôte comme l'a montré Upatham (1972), baisse de la température ralentissant le développement du parasite introduit, autres facteurs édaphiques. Il est impossible de faire la part exacte de ces différents facteurs qui interfèrent les uns avec les autres. Ainsi, lorsque la température baisse, le développement des miracidiums est ralenti, ce qui augmente d'autant la durée du séjour des œufs sur le fond avant l'éclosion, et par là la probabilité de leur

(3) La résistance à l'infestation qui se développe chez les individus âgés est un fait constaté expérımentalement; leur infestation reste cependant possible si l'on augmente le nombre de contacts Mollusque-miracidium par exposition à un grand nombre de miracidiums. 
ensevelissement. Une incubation préalable des œufs au laboratoire aurait peut-être permis de limiter les pertes par ensevelissement. Quant à la turbidité, il ne fait aucun doute qu'elle a joué un rôle très défavorable au cours de notre expérience ; la turbidité qui n'a jamais été inférieure à 100 J.T.U. est liée à la nature argileuse de la vase, laquelle est fréquemment mise en suspension, non seulement par le piétinement du bétail et des habitants, mais encore lors des fortes pluies. Les conditions régnant dans cette mare sont à opposer à celles qui règnent dans une autre localité de Guadeloupe, le Grand Etang, l'endémiotope naturel où la prévalence de $R$. marini s'est toujours avérée la plus forte (Nassi, 1978) ; ce milieu stagnant étudié par Pointier et al. (1977) est en particulier caractérisé par une faible turbidité (moins de 20 J.T.U.). Nous pensons que le nombre de rats utilisés aurait pu permettre de traiter une mare plus grande dans des conditions de turbidité plus favorables.

A partir des données numériques recueillies nous avons essayé d'estimer l'espérance de réussite des œufs introduits; nous avons choisi pour cela les données de l'automne 1976 et de l'automne 1977, périodes où la population comprend la plus forte proportion de Mollusques jeunes, donc réceptifs. A l'automne 1976, l'espérance de réussite a été de $1 / 200$; en 1977, pour un remplissage comparable de la mare mais avec une densité de Mollusques beaucoup plus faible, elle n'a été que de 1/1600. Bien entendu, compte tenu des erreurs d'échantillonnage, il n'est pas possible d'accorder à ces deux estimations d'autre valeur qu'indicative; elles montrent cependant que le rendement est faible.

Il apparaît donc que cet essai de contrôle est caractérisé à la fois par son succès, puisqu'il s'est soldé par la quasi-disparition des Mollusques, et par un rendement très faible de l'agent pathogène utilisé. Compte tenu des conditions défavorables dans lesquelles nous étions placés, liées notamment à la forte turbidité, nous pensons qu'on doit tirer de cette apparente contradiction un argument en faveur de la poursuite des recherches sur ce type de contrôle. Dans le cas de notre mare, l'efficacité de notre intervention aurait sans doute été considérablement améliorée par l'application d'un protocole mieux adapté aux caractéristiques démographiques de la population. Pour la mise en œuvre de ce protocole qui implique des interventions limitées dans le temps, mais massives, il pourrait s'avérer nécessaire de faire appel à des Trématodes stérilisants allochtones ayant une productivité en œufs supérieure à celle de $R$. marini guadeloupensis.

\section{REMERCIEMENTS}

Ce travail a reçu un support financier de la D.G.R.S.T. et de l'I.N.S.E.R.M. et a été réalisé dans le cadre de l'Action Concertée Bilharziose (Dir.: Y.-J. Golvan). La partie laboratoire a été réalisée en Guadeloupe à la Station de Zoologie de l'I.N.R.A. et dans le service de $\mathbf{M}^{\mathrm{me}}$ le $\mathrm{D}^{r} \mathrm{~S}$. Seytor (Hôpital de Pointe-à-Pitre).

\section{Bibliographie}

Heyneman (D.) et Umathevy (T.), 1967: A field experiment to test the possibility of using double infections of host snails as a possible biological control of schistosomoasis. Med. J. Malaya, 21,373 . 
Lie (K.J.), Kwo (E.H.) et Owyang (C.K.), 1970: A field trial to test the possible control of Schistosoma spindale by means of interspecific trematode antagonism. S. East Asian J. trop. Med. Pub. Hlth, 1, 19-28.

Lie (K.J.), Kwo (A.H.) et Owyang (C.K.), 1971: Further trial to control Schistosoma spindale by Trematode antagonism. S. East Asian J. trop. Med. Pub. Hlth, 2, 237-243.

Lie (K.J.) et Owyang (C.K.), 1973: A field trial to control Trichobilharzia brevis by dispersing eggs of Echinostoma audyi. S. East Asian J. trop. Med. Pub. Hlth, 4, 208-217.

Lie (K.J.), Schneider (C.R.), Sornmani (S.), Lanza (G.R.) et Impand (P.), $1974 a$ : Biological control by trematode antagonism. I. A successful trial to control Schistosoma spindale in northeast Thailand. S. East Asian J. trop. Med. Pub. Hlth, 5, 46-59.

Lie (K.J.), Schneider (C.R.), Sornmani (S.), Lanza (G.R.) et Impand (P.), 1974 b: Biological control by trematod antagonism. II. Failure to control Schistosoma spindale in a field trial in northeast Thailand. S. East Asian J. trop. Med. Pub. Hlth, 5, 60-64.

Nassi (H.), 1978: Données sur le cycle biologique de Ribeiroia marini guadeloupensis n.ssp. Trématode stérilisant Biomphalaria glabrata en Guadeloupe. Entretien du cycle en vue d'un contrôle éventuel des populations de Mollusques. Acta Trop., 35, 41-56.

Pointier (J.P.), Salvat (B.), Delplanque (A.) et Golvan (Y.J.), 1977: Principaux facteurs régissant la densité des populations de Biomphalaria glabrata (Say, 1818), Mollusque vecteur de la Schistosomose en Guadeloupe (Antilles françaises). Ann. Parasitol. Hum. Comp., 52, 277-323.

Upatham (E.S.), 1972: Effects of some physico-chemical factors on the infections of Biomphalaria glabrata (Say) by miracidia of Schistosoma mansoni Sambon in St Lucia, West Indies. J. Helminthol., 46, 307-315. 